\title{
BCL11A confers cell invasion and migration in androgen receptor-positive triple-negative breast cancer
}

\author{
XIN WANG $^{1 *}$, YUMEI XU ${ }^{2 *}, \mathrm{KUN} \mathrm{XU}^{3},{\text { YAJUAN } \mathrm{CHEN}^{4}, \text { XIUDI XIAO }}^{5}$ and XIAOXIANG GUAN ${ }^{1,3,4}$ \\ ${ }^{1}$ Department of Medical Oncology, Jinling Hospital, Medical School of Nanjing University, Nanjing, Jiangsu 210002; \\ ${ }^{2}$ Department of Gynaecology and Obstetrics, Zhongshan People's Hospital, Zhongshan, Guangdong 528403; \\ ${ }^{3}$ Department of Oncology, The First Affiliated Hospital of Nanjing Medical University, Nanjing, Jiangsu 210029; \\ ${ }^{4}$ Department of Medical Oncology, Jinling Clinical College of Nanjing Medical University, Nanjing, Jiangsu 210002; \\ ${ }^{5}$ Department of Breast Surgery, Affiliated People's Hospital of Jiangsu University, Zhenjiang, Jiangsu 212002, P.R. China
}

Received September 27, 2019; Accepted January 14, 2020

DOI: $10.3892 / 01.2020 .11383$

\begin{abstract}
Triple-negative breast cancer (TNBC) is associated with poor clinical prognosis due to a lack of effective therapeutic options. The expression of B-cell lymphoma/leukemia 11A (BCL11A) has been indicated to correlate with TNBC carcinogenesis, though the precise mechanisms of BCL11A-induced tumorigenesis in TNBC remain unclear. Using data retrieved from The Cancer Genome Atlas (TCGA) database, the present study demonstrated that BCL11A expression was upregulated in TNBC, compared with other types of breast cancer. Furthermore, in a tissue microarray of 140 patients with breast cancer, an elevated BCL11A level was correlated with unfavorable overall survival (OS), and exogenous BCL11A-knockdown was subsequently verified to inhibit tumor growth and metastasis in TNBC. Notably, the same tissue microarray revealed that a favorable patient outcome was associated with high expression levels of BCL11A and androgen receptor (AR). Moreover, BCL11 A-knockdown significantly inhibited the expression level of AR and further had an influence on proliferation, migration and invasion in
\end{abstract}

Correspondence to: Dr Xiaoxiang Guan, Department of Oncology, The First Affiliated Hospital of Nanjing Medical University, 300 Guangzhou Road, Nanjing, Jiangsu 210029, P.R. China

E-mail: xguan@nju.edu.cn

Dr Xiudi Xiao, Department of Breast Surgery, Affiliated People's Hospital of Jiangsu University, 8 Dianli Road, Zhenjiang, Jiangsu 212002, P.R. China

E-mail: xiaoxiudi@126.com

*Contributed equally

Abbreviations: BCL11A, B-cell lymphoma/leukemia 11A; TNBC, triple-negative breast cancer; TCGA, The Cancer Genome Atlas; OS, overall survival; DHT, dihydrotestosterone; HR, hazard ratio; HC, immunohistochemistry; ER, estrogen receptor; PR, progesterone receptor; AR, androgen receptor

Key words: BCL11A, TNBC, cell Invasion, cell migration, AR
TNBC cell lines. Collectively, the results of the current study indicate the function of BCL11A in TNBC progression, and provide new insights into the unique mechanism of BCL11A in AR regulation, emphasizing the significance of more research on BCL11A and AR regulation in TNBC molecular treatment.

\section{Introduction}

Breast cancer is an important public health threat to women worldwide. Triple-negative breast cancer (TNBC) accounts for $20 \%$ of total breast cancer cases, and is characterized as estrogen receptor (ER)-negative, progesterone receptor (PR)-negative, and HER2-negative (1). As a result of its aggressive biological characteristics and the lack of effective treatment options, TNBC has been associated with poor prognosis, when compared with other subtypes of breast cancer (2). Therefore, the development of novel TNBC therapies is urgently required. Recently, several studies have shown that other types of hormone receptors are expressed in TNBC, such as androgen receptor (AR) (3). AR was proved to have a high level of structural similarity to that of ER and PR (4). Several studies have demonstrated that different types of breast cancer exhibited diverse function of AR. AR-associated signaling pathway was speculated to act as either tumorigenic or antitumor effect in multiple studies in breast cancer. A previous study identified AR as a commonly expressed nuclear receptor in breast cancer, which was crucial in promoting tumorigenesis (5). In ER-positive breast cancer, AR could predict promising outcome, while in TNBC, the prognostic value of AR expression is still controversial. In TNBC, multiple researches have illustrated different results on the function of AR (5-8). Furthermore, it has been found that bicalutamide, an AR inhibitor, could reduce TNBC proliferation and activity (1). Nevertheless, the precise molecular mechanisms of AR-induced cell proliferation, migration and invasion in TNBC cells remains unclear. The present study identified BCL11A, a novel oncogene, and its potential role in the AR-associated regulation of TNBC.

BCL11A, also known as Evi9 and CTIP1, was firstly discovered in leukemia with chromosome translocation $\mathrm{t}(2 ; 14)(\mathrm{p} 13 ; \mathrm{q} 32.3)$. The BCL11 A gene is located on human 
chromosome 2 p13 and is $\sim 102 \mathrm{~kb}$ in length (9). BCL11A is primarily expressed in brain and hematopoietic tissues (10), though accumulating evidence suggests that it also plays an essential role in the formation of other tumors, including prostate cancer, lung cancer, laryngeal squamous cell carcinoma and acute leukemia (11-16). In lung cancer, Jiang et al identified BCL11A could induced by miR-30a and act as a potential prognostic factor in NSCLC (17). Additionally, in lung squamous cell carcinoma (LUSC), Lazarus et al found that BCL11A was integral to LUSC pathology and could interact with SOX2 to regulate several downstream transcription factors (18). In prostate cancer and colorectal cancer, it has been demonstrated that BCL11A overexpression strongly reversed the influence of tumor progression induced by FOXQ1 inhibition $(19,20)$. Notably, BCL11A may also promote stemness in breast cancer cells by inducing Wnt/ $\beta$-catenin signaling (21). BCL11A activation is associated with the induction of numerous carcinogenic signaling pathways, and there is growing evidence to suggest that BCL11A downregulation inhibits tumorigenesis $(22,23)$. However, the specific functions of BCL11A and the underlying mechanism of its involvement in TNBC have not been fully clarified. The results of the present study demonstrated the importance of BCL11A in the development and progression of TNBC, and for the first time, identified the potential mechanism of BCL11A-associated AR regulation in TNBC.

\section{Materials and methods}

Clinical samples. Tissue microarray HBre-Ducl40Sur-01 was obtained from Shanghai Outdo Biotech Co., Ltd, and contained tissue samples collected between January 2001 and August 2004. The corresponding patients had not received radiotherapy or chemotherapy prior to surgery. Experiments with tumor tissues were performed according to the Declaration of Helsinki and were approved by the Ethics Committee of Jinling Hospital. Construction of the tissue microarray has previously been demonstrated (24). The surgical time was between 2001.07-2004.08. The follow-up time was up to 2013.07. The median survival time was 76 months.

TCGA database. Breast cancer tissue samples with RNA-seq data and clinicopathological information were downloaded from the cancer genome atlas (TCGA) database released on October 31, 2018. Samples were divided into four breast cancer subtypes according to their ER, PR and HER2 status. At the time of analysis, 466 luminal A samples, 103 luminal B samples, 39 HER2-positive samples and 140 TNBC samples were included in our study. Overall survival (OS) was measured from the date of diagnosis to the date of death due to all causes or the last follow-up.

Immunohistochemistry. Immunohistochemical staining was performed as previously described (24). Staining was scored as: i) ++++ if $>80 \%$ tumor cells were immuno-positive; ii) +++ for $51-80 \%$; iii) ++ for $11-50 \%$; iv) + for $1-10 \%$ cells; and v) -if $<1 \%$ of the tumor cells were positively stained. The staining intensity was categorized as negative (-), weak (+), moderate $(++)$ or strong $(+++)$. The $\mathrm{H}$-score method was utilized via multiplying the percentage score by the staining intensity score and produce a summed score between 0 and 12 . The standard of classification was shown as follows: Intensity score $(0=$ no staining, $1=$ weak, $2=$ moderate and $3=$ strong); stained cell proportion score $(0=0 \%, 1=1-10,2=11-50,3=51-80$ and $4=81-100 \%$ ). BCL11A high expression was defined as $H$ score $\geq 6$. AR positive was defined as $\geq 10 \%$ expression. Immunostained sections were observed using a microscope (Carl Zeiss Inc.).

Cell lines and culture conditions. TNBC cell lines (MDA-MB-231 and Hs578T) were provided by Chinese Academy of Science Committee Type Culture Collection Cell Bank (Shanghai, China). Cell culture experiment was conducted as recently described (25). MDA-MB-231 and Hs578T cells were cultured in RPMI 1640 and DMEM medium, respectively, both supplemented with $10 \%$ fetal bovine serum (FBS) and 100 units/ml penicillin/streptomycin (all Gibco; Thermo Fisher Scientific, Inc.). The cells were maintained at $37^{\circ} \mathrm{C}$ with $5 \% \mathrm{CO}_{2}$.

Plasmids and transient transfection. Short hairpin (sh)RNA sh-BCL11A and the negative control sh-NC were cloned into expression plasmids to generate recombinant lentiviral vectors (LV-shRNA-BCL11A and LV-shRNA-NC). Cells were transfected with recombinant lentivirus plasmid LV-shRNA-BCL11A or LV-shRNA-NC in the presence of $5 \mu \mathrm{g} / \mu \mathrm{l}$ polyene (Sigma-Aldrich; Merck KGaA). Lentivirus plasmids were provided by Suzhou GenePharma Co., Ltd. and si-BCL11A was provided by Guangzhou RiboBio Co., Ltd. The sequences were: sh-BCL11A: 5'-GCAGATAAACTTCTG CACTGG-3'; sh-NC: 5'-TTCTCCGAACGTGTCACGT-3'; si-BCL11A: 5'-GAACACTCATGGATTAAGA-3'.

Western blotting. Samples were prepared using a RIPA lysis buffer containing a protease inhibitor cocktail. The protein concentrations were quantified with the BCA kit (All kits from KeyGen Biotech). Subsequent experiment was conducted as recently described (26). The antibodies used for western blotting were as follows: Rabbit anti-BCL11A (ab191401, 1:10,000, Abcam), rabbit anti-AR (ab74272, 1:300, Abcam), rabbit anti-GAPDH (ab181603, 1:10,000, Abcam) and rabbit IgG (\#7074, 1:10,000, Cell Signaling Technologies, Inc.).

Colony formation assay. Transfected cells were seeded into a 6-well plate (600 cells per well) and incubated in fresh medium at $37^{\circ} \mathrm{C}$ for 14 days. The resulting colonies were fixed with $100 \%$ methanol. And then colonies were stained with $0.5 \%$ crystal violet. Colonies containing $>50$ cells were manually numbered.

Wound-healing assay. Following transfection, cells were seeded into 6-well plates and incubated at $37^{\circ} \mathrm{C}$ until $90 \%$ confluence. A sterile pipette tips was used to create a wound across each cell monolayer, and the cells were incubated in serum-free medium for a further $12 \mathrm{~h}$. Images of the wound area were captured at 0 and $12 \mathrm{~h}$, and wound closure was assessed using Image-Pro Plus 6.0 (Media Cybernetics, Inc.).

Transwell assay. Following transfection, $2 \times 10^{4}$ cells (in $200 \mu 1$ serum-free medium) were added to the upper chamber of a 

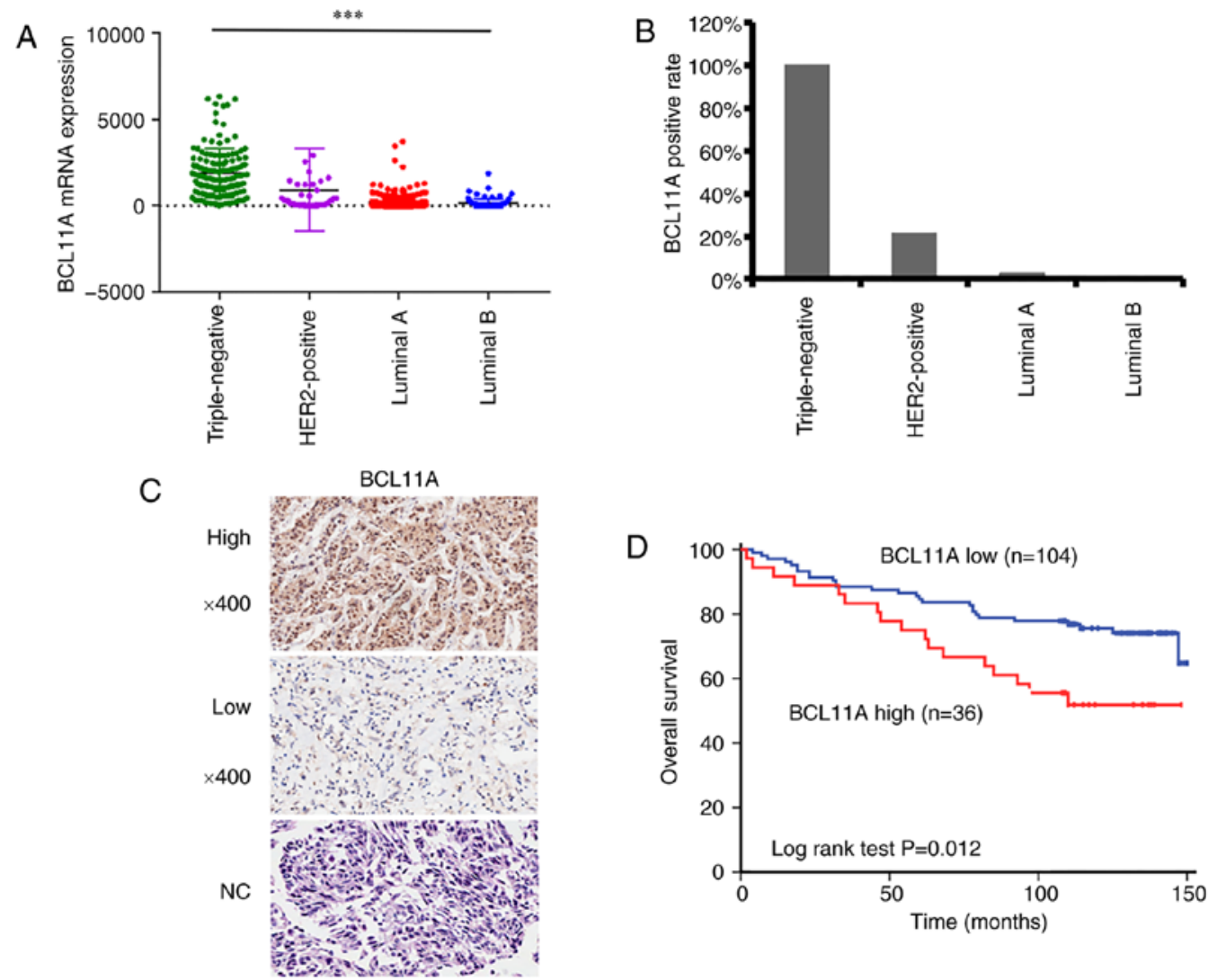

Figure 1. BCL11A expression in triple-negative breast cancer tissues and its association with survival. (A) BCL11A mRNA expression data in different subtypes of breast cancer were extracted from The Cancer Genome Atlas mRNA database. One-way ANOVA analysis was performed among four groups. ${ }^{* * * *} \mathrm{P}<0.001$. (B) BCL11A expression in different subtypes of breast cancer using a tissue microarray is presented. (C) Representative immunohistochemical staining images of BCL11A protein level in patient tissues are presented. Patients were divided into high (image above) and low (image below) BCL11A expression groups according to the histochemistry score. (D) Overall survival of patients with breast cancer with high (red) or low (blue) BCL11A expression is presented. $\mathrm{P}=0.012$. $\mathrm{BCL} 11 \mathrm{~A}, \mathrm{~B}$-cell lymphoma/leukemia $11 \mathrm{~A} ; \mathrm{NC}$, negative control.

Transwell insert; $800 \mu 1$ of medium (supplemented with $10 \%$ FBS) was added to the lower chamber as a chemoattractant. After culturing at $37^{\circ} \mathrm{C}$ for $24 \mathrm{~h}$, cells that had migrated to the lower matrix membrane were stained with crystal violet; stained cells were counted using a light microscope.

Cellcycle analysis. Cells transfected withLV-shRNA-BCL11A, LV-shRNA-NC or the mock were cultured in 6-well plates at $2 \times 10^{5}$ per well. Cells were harvested and centrifuged at $1500 \mathrm{rpm}$ for $5 \mathrm{~min}$. Subsequent steps were performed as recently described (27). The cells were washed with ice-cold PBS and fixed with $70 \%$ ethanol overnight at $-20^{\circ} \mathrm{C}$. The fixed cells were washed with PBS for 10 min and treated with RNAase A for 30 min followed by incubation with propidium iodide for $30 \mathrm{~min}$ at room temperature. The cell cycle in each specific sample were evaluated by the flow cytometer (FACSCalibur) following the manufacture's instruction. The ModFit software was used to analyze the cell cycle result.

Statistical analysis. Data analysis was performed with SPSS v.20 (IBM Corp.) and GraphPad Prism software v.7 (GraphPad Software, Inc.). All experiments were independently conducted $\geq 3$ times. Comparisons between two groups were analyzed using Student's t-test. Comparisons between multiple groups were analyzed using one-way ANOVA, followed by Tukey's post hoc test. For the IHC results, P-values were calculated using the $\chi^{2}$ test to compare the distribution of demographic variables. Survival analysis were performed according to Kaplan-Meier method. Prognostic factor was calculated using Cox proportional hazards regression model. $\mathrm{P}<0.05$ was considered to indicate a statistically significant difference.

\section{Results}

Elevated expression of BCL11A correlates with an unfavorable outcome of breast cancer patients. To evaluate the expression level of BCL11A in breast cancer, BCL11A mRNA expression data was extracted from TCGA database, which showed that TNBC tumors expressed significantly increased levels of BCL11A compared with those of other types of breast cancer $(\mathrm{P}<0.001)($ Fig. 1A). Subsequently, IHC staining was performed in a breast cancer microarray (140 tissue samples) to detect the expression of BCL11A (Fig. 1B-C). The positive rates of BCL11A in TNBC, HER-2 breast cancer and luminal A breast cancer were $100 \%$ (31/31), 21.4\% (2/14) and $2.9 \%(2 / 70)$, respectively. Demographic, pathological and clinical variables were also analyzed. Furthermore, the association between BCL11A expression and clinical characteristics of 140 cases was reviewed and analyzed (Table I). Results showed that 36 cases (25.7\%) exhibited high BCL11A levels, while 104 cases (74.3\%) exhibited low BCL11A levels. 
Table I. Relationship between expression of BCL11A and clinicopathologic features of patients with breast cancer.

\begin{tabular}{|c|c|c|c|c|c|c|}
\hline \multirow[b]{2}{*}{ Variables } & \multicolumn{2}{|c|}{$\begin{array}{l}\text { BCL11A high } \\
\quad(n=36)\end{array}$} & \multicolumn{2}{|c|}{$\begin{array}{l}\text { BCL11A low } \\
(\mathrm{n}=104)\end{array}$} & \multirow[b]{2}{*}{$\chi^{2}$} & \multirow[b]{2}{*}{ P-value } \\
\hline & $\mathrm{n}$ & $\%$ & $\mathrm{n}$ & $\%$ & & \\
\hline Age & & & & & 0.946 & 0.331 \\
\hline$\leq 51$ years & 16 & 44.4 & 56 & 53.8 & & \\
\hline$>51$ years & 20 & 55.6 & 48 & 46.2 & & \\
\hline Histological $^{\mathrm{a}}$ & & & & & 3.278 & 0.166 \\
\hline I & 1 & 2.8 & 11 & 10.9 & & \\
\hline II-III & 35 & 97.2 & 90 & 89.1 & & \\
\hline TNM stage $^{\mathrm{b}}$ & & & & & 0.714 & 0.700 \\
\hline I-II & 24 & 66.7 & 67 & 65.7 & & \\
\hline III & 12 & 33.3 & 35 & 34.3 & & \\
\hline ER status ${ }^{c}$ & & & & & 80.545 & $<0.001$ \\
\hline Negative & 34 & 94.4 & 13 & 13.5 & & \\
\hline Positive & 2 & 5.6 & 83 & 86.5 & & \\
\hline PR status ${ }^{\mathrm{d}}$ & & & & & 62.177 & $<0.001$ \\
\hline Negative & 35 & 97.2 & 23 & 24.0 & & \\
\hline Positive & 1 & 2.8 & 73 & 76.0 & & \\
\hline HER-2 status ${ }^{\mathrm{e}}$ & & & & & 9.503 & 0.009 \\
\hline Negative & 33 & 91.7 & 68 & 70.8 & & \\
\hline Positive & 3 & 8.3 & 28 & 29.2 & & \\
\hline AR status ${ }^{\mathrm{f}}$ & & & & & 4.223 & 0.040 \\
\hline Negative & 8 & 22.2 & 43 & 41.3 & & \\
\hline Positive & 28 & 77.8 & 61 & 58.7 & & \\
\hline
\end{tabular}

In addition, these data revealed that BCL11A expression was not significantly correlated with age, histological or TNM stage. Notably, a high BCL11A level was strongly associated with decreased level of ER, PR and HER-2, and an increased level of AR. Then, correlation between BCL11A level with patient clinical outcomes was investigated, revealing that high BCL11A expression was correlated with poor OS (Fig. 1D, $\mathrm{P}=0.012$ ). In order to elucidate whether OS was correlated with any of the clinicopathological variables, multivariate analysis with Cox proportional hazard model was applied. As displayed in Table II, several factors were significantly associated. Therefore, BCL11A was speculated to act as an independent unfavorable prognostic biomarker in breast cancer $[(\mathrm{HR})=2.099 ; 95 \%(\mathrm{CI})=1.123-3.925 ; \mathrm{P}=0.020]$. In summary, these results suggest that in breast cancer patients, BCL11A expression is correlated with a poor outcome.

BCL11A-knockdown reduces TNBC cell proliferation. Multiple researches have demonstrated that BCL11A is critically associated with tumor development and progression.
However, the function of BCL11A in breast cancer, particularly in TNBC, requires additional in-depth exploration. The present study demonstrated that TNBC tissues exhibited significantly higher BCL11A expression compared with other types of breast cancer. To further gain evidence of its crucial role in TNBC, the effects of BCL11A inhibition on the tumorigenic capacity of TNBC cells were investigated, using an shRNA-knockdown assay (Fig. 2A). BCL11A expression was not significantly related with cell cycle (Fig. 2B, Fig. S1A-F). However, the results of the colony formation assay showed that BCL11A-knockdown significantly reduced the cell proliferative ability in both cell lines (Fig. 2C). These data thus indicated that BCL11A could induce TNBC cell proliferation and promote cancer development and progression.

BCL11A induces TNBC cell migration and invasion. Few studies have elucidated the correlation between BCL11A expression and cell proliferation in TNBC. The present study indicated that BCL11A promoted TNBC proliferation. To further evaluate its biological function, the influence of 
Table II. Univariate Cox proportional hazard regression model analysis of breast cancer for overall survival.

\begin{tabular}{|c|c|c|c|c|}
\hline \multirow[b]{2}{*}{ Variable } & \multicolumn{2}{|c|}{ Univariate analysis } & \multicolumn{2}{|c|}{ Multivariate analysis } \\
\hline & $\mathrm{HR}(95 \% \mathrm{CI})$ & P-value & $\mathrm{HR}(95 \% \mathrm{CI})$ & P-value \\
\hline Age & $1.397(0.769-2.538)$ & 0.272 & & \\
\hline TNM stage & $2.361(1.307-4.25)$ & 0.004 & $2.543(1.398-4.625)$ & 0.002 \\
\hline \multicolumn{5}{|l|}{ Tumor size } \\
\hline$\leq 2 \mathrm{~cm}$ & 1.000 & & 1.000 & \\
\hline $2-5 \mathrm{~cm}$ & $1.403(0.617-3.10)$ & 0.419 & $1.237(0.541-2.828)$ & 0.614 \\
\hline$>5 \mathrm{~cm}$ & $2.355(0.786-7.01)$ & 0.126 & $1.584(0.478-5.251)$ & 0.452 \\
\hline \multicolumn{5}{|l|}{ Node status } \\
\hline 0 & 1.000 & & 1.000 & \\
\hline $1-3$ & $1.003(0.439-2.29)$ & 0.994 & $0.897(0.381-2.113)$ & 0.840 \\
\hline $4-9$ & $2.454(1.190-5.01)$ & 0.015 & $2.464(0.231-26.27)$ & 0.455 \\
\hline$\geq 10$ & $2.177(0.709-6.64)$ & 0.174 & $2.474(0.212-28.93)$ & 0.470 \\
\hline Histological & $0.953(0.341-2.67)$ & 0.928 & & \\
\hline ER status & $0.532(0.288-0.984)$ & 0.044 & $0.597(0.314-1.134)$ & 0.115 \\
\hline PR status & $0.528(0.284-0.99)$ & 0.043 & $0.599(0.313-1.146)$ & 0.121 \\
\hline HER-2 status & $0.829(0.382-1.76)$ & 0.634 & $0.718(0.324-1.592)$ & 0.415 \\
\hline BCL11A status & $2.150(1.169-3.96)$ & 0.014 & $2.099(1.123-3.925)$ & 0.020 \\
\hline
\end{tabular}

Bold values are statistically significant $(\mathrm{P}<0.05)$. ER, estrogen receptor; PR, progesterone receptor; HER-2, human epidermal growth factor receptor-2; BCL11A, B-cell lymphoma/leukemia 11A.

A

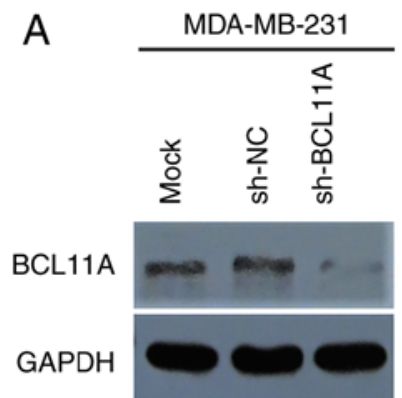

C
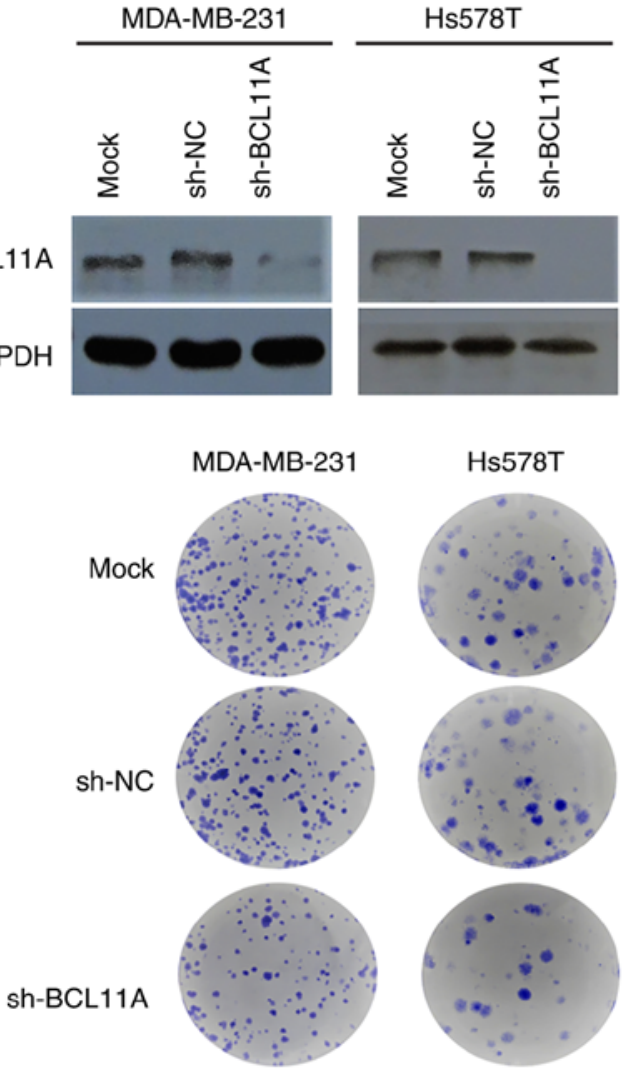

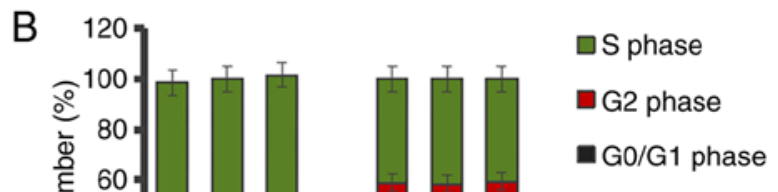

言

焉 40

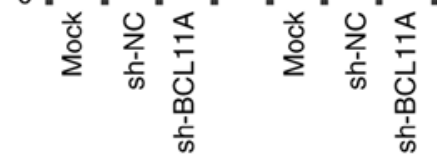

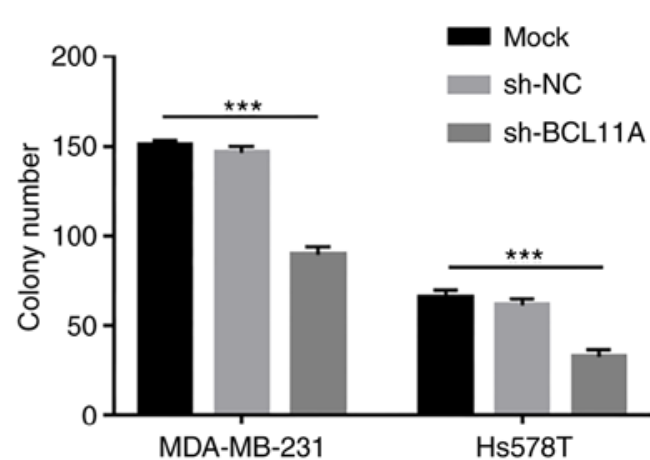

Figure 2. BCL11A-knockdown reduces triple-negative breast cancer cell proliferation. (A) Western blot analysis of BCL11A expression after knockdown with LV-sh-BCL11A in MDA-MB-231 and Hs578T cells were conducted. Representative blots are presented. (B) Flow cytometry was used to detected the percentage of G0/G1, S and G2 phase of MDA-MB-231 and Hs578T cells after LV-sh-BCL11A infection. Green, red and black bars represent cells in the S, G2 and G0/G1 phase, respectively. (C) Proliferative ability of MDA-MB-231 and Hs578T cells was detected by the colony formation analysis after infection with LV-sh-BCL11A. Colony numbers were counted and were compared among the mock group, sh-NC group and sh-BCL11A group. ${ }^{* * *} \mathrm{P}<0.001$. BCL11A, B-cell lymphoma/leukemia 11A; sh, short hairpin; NC, negative control; LV, lentivirus. 

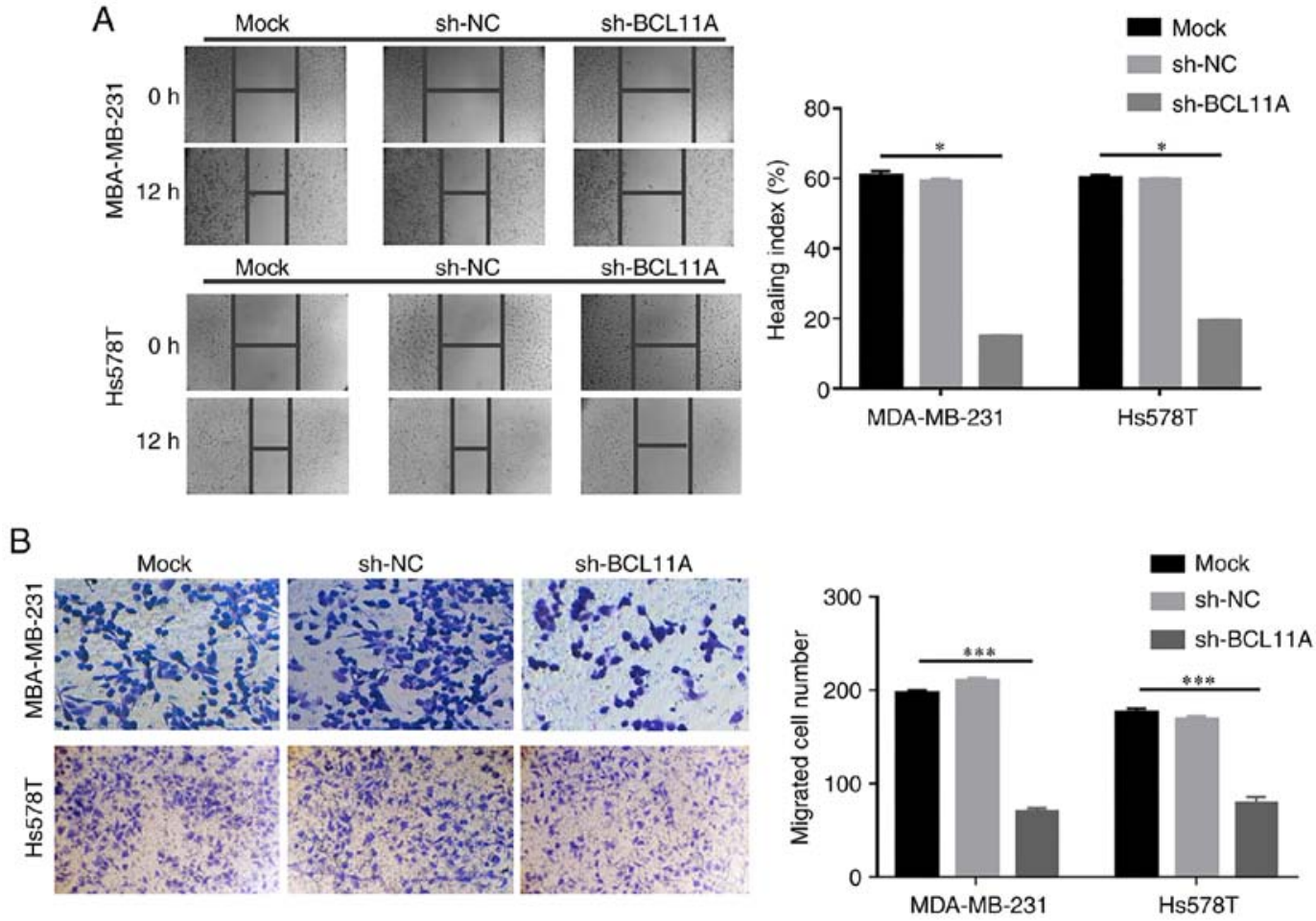

Figure 3. BCL11 A promotes triple-negative breast cancer cell migration and invasion. (A) Wound healing assay was applied to measure the migratory ability of MDA-MB-231 and Hs578T cells after infection with LV-sh-BCL11A. (B) Transwell analysis was conducted to measure the invasive ability of MDA-MB-231 and Hs578T cells after infection with LV-sh-BCL11A. Cells were added to transwell inserts and allowed to migrate for $24 \mathrm{~h}$. Representative images are presented. Magnification, $\mathrm{x} 100 .{ }^{*} \mathrm{P}<0.05,{ }^{* * * *} \mathrm{P}<0.001$. BCL11A: B-cell lymphoma/leukemia 11A; sh, short hairpin; NC, negative control; LV, lentivirus.

A

\begin{tabular}{cc|c}
\hline & \multicolumn{2}{c}{$\begin{array}{c}\text { Testing set } \\
\text { Tumor microarray }(\mathrm{n}=140)\end{array}$} \\
\cline { 2 - 3 } & AR positive & AR negative \\
\hline BCL11A (high) & 28 & 8 \\
BCL11A (low) & 61 & 43 \\
\hline $\mathrm{r}$ & \multicolumn{2}{c}{0.174} \\
$\mathrm{p}$ & \multicolumn{2}{c}{0.040} \\
\hline
\end{tabular}

C

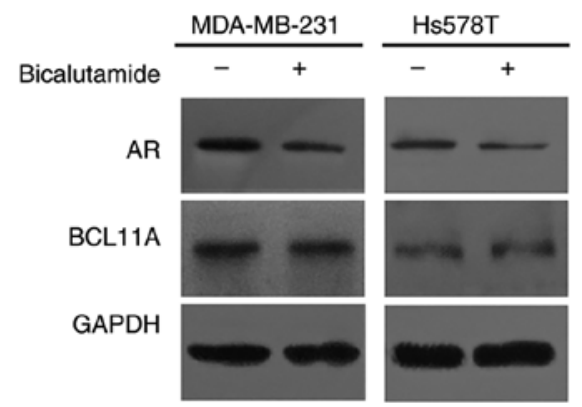

B

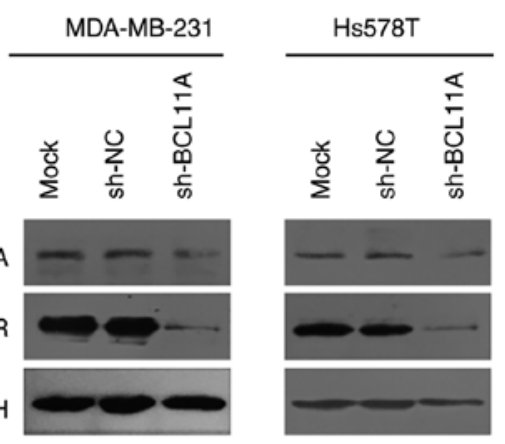

D

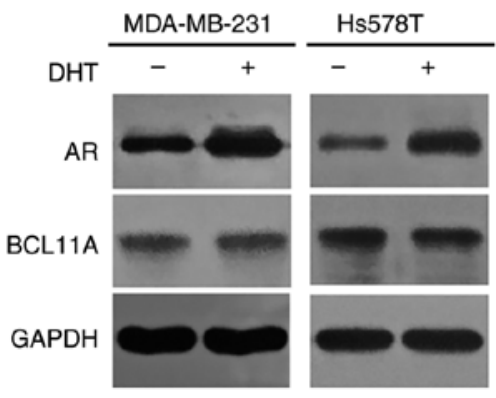

Figure 4. BCL11A-knockdown expression downregulates the AR level in the triple-negative breast cancer cells. (A) Correlation analysis between BCL11A expression and $\mathrm{AR}$ using 140 breast cancer samples in a microarray. P-value was determined using Cox proportional hazards regression model. $\mathrm{P}=0.040$. (B) Western blot analysis was applied to detect AR expression in MDA-MB-231 and Hs578T cells after BCL11A-knockdownWestern blot analysis was used for detection of BCL11A protein level in MDA-MB-231 and Hs578T cells following treatment with (C) bicalutamide (10 $\mu$ M in DMSO) and (D) DHT (100 nM in ethanol) and for 48 h. BCL11 A, B-cell lymphoma/leukemia 11A; AR, androgen receptor; DHT, dihydrotestosterone; sh, short hairpin; NC, negative control.

BCL11A protein on TNBC migration and invasiveness ability was determined. Using a wound-healing assay, BCL11A knockdown noticeably inhibited cell migration in both cell lines (Fig. 3A). Likewise, a Transwell assay revealed that BCL11A-knockdown significantly decreased the metastatic potential of TNBC cells (Fig. 3B). These data thus revealed that 


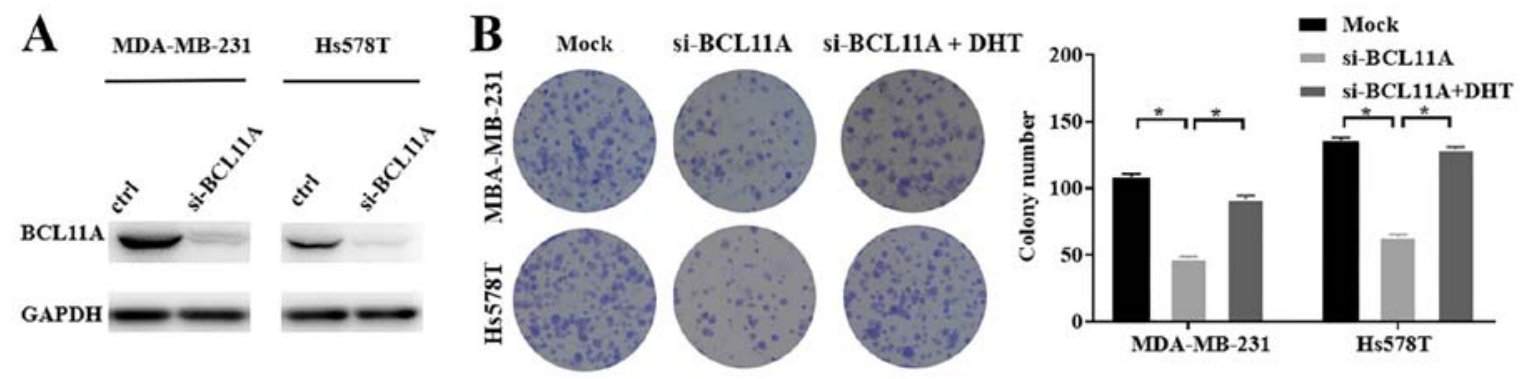

C
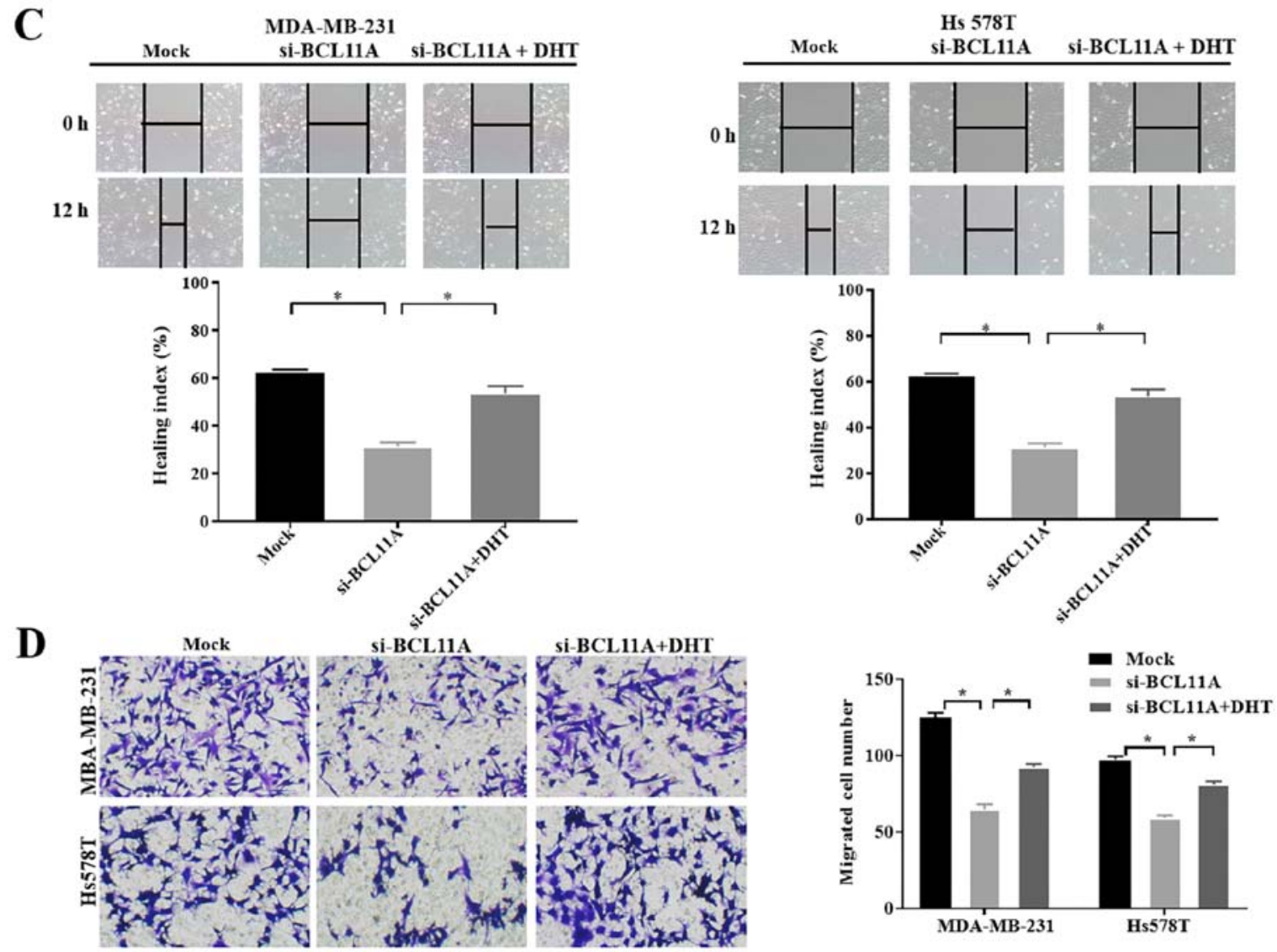

Figure 5. DHT significantly reverses the antitumor effect in BCL11A-knockdown triple-negative breast cancer cells. (A) Western blot analysis of BCL11A expression after knockdown with si-BCL11A in MDA-MB-231 and Hs578T cells were conducted. Representative blots are presented. (B) Proliferative ability of MDA-MB-231 and Hs578T cells was detected by the colony formation analysis after infection with si-BCL11A and DHT. Colony numbers were counted and were compared among the mock group, si-BCL11A group and si-BCL11A + DHT group. (C) Wound healing assay was applied to measure the migratory ability of MDA-MB-231 and Hs578T cells after infection with si-BCL11A and DHT. (D) Transwell analysis was conducted to measure the invasive ability of MDA-MB-231 and Hs578T cells after infection with si-BCL11A and DHT. Cells were added to transwell inserts and allowed to migrate for $24 \mathrm{~h}$. Representative images are presented. Magnification, x1100. ${ }^{*} \mathrm{P}<0.05$. BCL11A, B-cell lymphoma/leukemia 11A; si, small interfering; DHT, dihydrotestosterone.

BCL11A could facilitate TNBC migration and invasion, which may result in carcinogenesis and metastasis. Collectively, these results confirmed that exogenous BCL11A knockdown inhibited tumor progression in TNBC, and support the tumorigenic role of BCL11A in TNBC cell function.

$B C L 11$ A regulates the expression of AR in TNBC cells. The aforementioned data showed that BCL11 A was upregulated and served as a carcinogenic factor in TNBC. Although TNBC is characterized as lacking of ER, PR and HER-2, it expresses other receptors like AR. Yet the correlation of BCL11A and AR in TNBC cells remains unknown. To address it, the present study evaluated if inhibiting BCL11A could influence AR expression level in TNBC cell lines. Using a tissue microarray, a significant positive association was illustrated between
BCL11A level and AR (Fig. 4A). Furthermore, knockdown of BCL11A expression significantly down-regulated the expression level of AR in TNBC cells (Fig. 4B). To determine whether the AR reversely regulated BCL11A expression, TNBC cells were treated for $48 \mathrm{~h}$ with dihydrotestosterone (DHT), an AR agonist, or bicalutamide, an AR antagonist. BCL11A protein expression was then analyzed by western blotting. The results show that the activation or inhibition of AR signaling had no significant impact on BCL11A protein expression level (Fig. 4C and D). Moreover, in the functional assay, the AR agonist, DHT, significantly reversed the antitumor effect of si-BCL11A in both TNBC cell lines (Fig. 5A-D). Collectively, these results provided solid evidence that BCL11A could induce the expression of AR and further had an influence on proliferation, migration and invasion in TNBC cell lines. 


\section{Discussion}

In the current study, BCL11A was shown to be upregulated, and to be a predictor of poor clinical outcome in patients with TNBC. BCL11A was determined to serve as an independent indicator of unfavorable outcome in breast cancer. Experimentally, down-regulating BCL11A expression in TNBC cells significantly inhibited tumor progression. Statistical analysis demonstrated that high BCL11A level was associated with higher expression of AR. Subsequent experimentation also revealed that knockdown of BCL11A expression significantly down-regulated the expression level of AR in TNBC cell lines and further had an influence on cell function in TNBC.

It has previously been disclosed that as well as normal lymphoid development and globin switching, BCL11A plays an active role in mediating tumorigenesis $(28,29)$. In bone metastasis of breast cancer, the expression of BCL11A is significantly increased compared with control group (30). In the present study, BCL11A was shown to be upregulated in patients with TNBC, and high BCL11A protein level was associated with poor OS, suggesting that there is a correlation of BCL11A expression with breast cancer. Regarding tumorigenesis in prostate cancer, it was previously shown that BCL11A overexpression strongly reversed the influence of tumor progression induced by FOXQ1 inhibition (19). On the basis of previous results, in TNBC cells, disrupting BCL11A expression significantly reduced cell proliferation and invasion. Additionally, gene expression profiling of BCL11A siRNA-treated SUDHL6 cells proved that BCL11A could be associated with the signaling network of cell cycle (22). However, the present data did not indicate a relationship between BCL11A and cell cycle in TNBC. The possible reason may be that BCL11A cannot increase transcriptional rate in TNBC, which would lead to the decreased expression of cyclin genes. Tsang et al investigated the cell cycle phenotype in the BCL11A-deficiency hematopoietic stem cells (HSCs), suggesting the reduction of quiescence in the $\mathrm{Bcllla}^{-/-} \mathrm{HSC}$ compartment (31). However, changes in the transcriptome are one of the earliest events in entry to the cell cycle. Therefore, the cell cycle progression requires further translational and post-translational regulation, which might accout for our cell cycle result. During neocortical development, BCL11A is necessary for the cell polarity switch and upper layer neuron migration (32). In current study, disrupting BCL11A expression significantly reduced cell migration in TNBC. Finally, our data provided compelling evidence that BCL11A is critical in the tumor progression of TNBC.

In addition, it is important to elucidate the potential underlying mechanism in TNBC and the downstream targets of BCL11A in TNBC. As we all known, TNBC is characterized as lacking of ER, PR and HER-2. However, it expresses other hormone receptors like AR. AR has been recognized to act as a very important factor in recent years. Our present data indicated that high BCL11A level correlated with higher level of AR in breast cancer patients. Although correlation coefficient $r$ is only 0.174 , we evaluated to focus on the significance of P-value, which was 0.040 and statistically significant. Previous study has reported that AR was expressed in a particular group of TNBC patients and was strongly correlated with clinical prognosis. Increasing evidence has indicated that AR signaling pathway played a critical role in modulating oncogenesis and tumor metastasis. To gain more solid evidence, we designed the following experiment in TNBC cell lines to further investigate if inhibiting BCL11A could influence AR expression level and subsequently influence tumor cell function. The present study showed that BCL11A knockdown significantly down-regulated the expression level of AR in TNBC cells and further had an influence on cell function, which suggests that AR may be a downstream target of BCL11A. Also, the results of DHT or bicalutamide treatment in TNBC cells demonstrated that activating or inhibiting AR signaling had no significant impact on BCL11A protein level. To the best of our knowledge, the present study is the first to reveal a unique molecular mechanism by which BCL11A regulates the level of $\mathrm{AR}$ in TNBC, and further research in the future is required to determine the precise mechanism of BCL11A and AR in TNBC tumorigenesis and potential targeted therapies.

In summary, the present study suggests that BCL11A as a novel biomarker of tumorigenesis, which acts through the upregulation of the AR in breast cancer. Therefore, BCL11A may act as both a new prognostic predictor and a feasible target for TNBC therapy. Moreover, these data indicate that in-depth research of BCL11A and AR as promising implications for TNBC-targeted therapeutic options is warranted.

\section{Acknowledgements}

Not applicable.

\section{Funding}

The present study was supported by The National Natural Science Foundation of China (grant no. 81773102) and The Key International Cooperation of the National Natural Science Foundation of China (grant no. 81920108029 to XXG).

\section{Availability of data and materials}

The datasets used and/or analyzed during the current study are available from the corresponding author on reasonable request.

\section{Authors' contributions}

XW and YX performed the experiments, wrote the main manuscript and analyzed the data. KX performed the experiments. YC helped with the statistical analysis. XG and XX designed the project and revised the manuscript. All authors contributed to the revised manuscript. All authors read and approved the final manuscript.

\section{Ethics approval and consent to participate}

The present study was approved by The Ethics Committee of Jinling Hospital. Written informed consent was obtained from each patient.

\section{Patient consent for publication}

Not applicable. 


\section{Competing interests}

The authors declare that they have no competing interests.

\section{References}

1. Lehmann BD, Bauer JA, Chen X, Sanders ME, Chakravarthy AB, Shyr Y and Pietenpol JA: Identification of human triple-negative breast cancer subtypes and preclinical models for selection of targeted therapies. J Clin Invest 121: 2750-2767, 2011.

2. Millikan RC, Newman B, Tse CK, Moorman PG, Conway K, Dressler LG, Smith LV, Labbok MH, Geradts J, Bensen JT, et al: Epidemiology of basal-like breast cancer. Breast Cancer Res Treat 109: 123-139, 2008.

3. Skor MN, Wonder EL, Kocherginsky M, Goyal A, Hall BA, Cai Y and Conzen SD: Glucocorticoid receptor antagonism as a novel therapy for triple-negative breast cancer. Clin Cancer Res 19: 6163-6172, 2013.

4. Lonergan PE and Tindall DJ: Androgen receptor signaling in prostate cancer development and progression. J Carcinogenesis 10 20, 2011.

5. Mrklic I, Pogorelic Z, Capkun V and Tomić S: Expression of androgen receptors in triple negative breast carcinomas. Acta Histochem 115: 344-348, 2013.

6. Vera-Badillo FE, Templeton AJ, de Gouveia P, Diaz-Padilla I, Bedard PL, Al-Mubarak M, Seruga B, Tannock IF, Ocana A and Amir E: Androgen receptor expression and outcomes in early breast cancer: A systematic review and meta-analysis. J Nat Cancer Inst 106: djt319, 2014.

7. He J, Peng R, Yuan Z, Wang S, Peng J, Lin G, Jiang X and Qin T: Prognostic value of androgen receptor expression in operable triple-negative breast cancer: A retrospective analysis based on a tissue microarray. Med Oncol 29: 406-410, 2012.

8. Gerratana L, Basile D, Buono G, De Placido S, Giuliano M, Minichillo S, Coinu A, Martorana F, De Santo I, Del Mastro L, et al: Androgen receptor in triple negative breast cancer: A potential target for the targetless subtype. Cancer Treat Rev 68: 102-110, 2018.

9. Satterwhite E, Sonoki T, Willis TG, Harder L, Nowak R, Arriola EL, Liu H, Price HP, Gesk S, Steinemann D, et al: The BCL11 gene family: Involvement of BCL11A in lymphoid malignancies. Blood 98: 3413-3420, 2001.

10. Saiki Y, Yamazaki Y, Yoshida M, Katoh O and Nakamura T: Human EVI9, a homologue of the mouse myeloid leukemia gene, is expressed in the hematopoietic progenitors and down-regulated during myeloid differentiation of HL60 cells. Genomics 70: 387-391, 2000

11. Jin $\mathrm{C}$, Yu W, Lou X, Zhou F, Han X, Zhao N and Lin B UCHL1 is a putative tumor suppressor in ovarian cancer cells and contributes to cisplatin resistance. J Cancer 4: 662-670, 2013.

12. Boelens MC, Kok K, van der Vlies P, van der Vries G, Sietsma H, Timens W, Postma DS, Groen HJ and van den Berg A: Genomic aberrations in squamous cell lung carcinoma related to lymph node or distant metastasis. Lung Cancer 66: 372-378, 2009.

13. Podgornik H, Pretnar J, Skopec B, Andoljšek D and Černelč P: Concurrent rearrangements of BCL2, BCL3, and BCL11A genes in atypical chronic lymphocytic leukemia. Hematology 19 $45-48,2014$.

14. Kapatai G and Murray P: Contribution of the Epstein Barr virus to the molecular pathogenesis of Hodgkin lymphoma. J Clin Pathol 60: 1342-1349, 2007.

15. Chetaille B, Bertucci F, Finetti P, Esterni B, Stamatoullas A, Picquenot JM, Copin MC, Morschhauser F, Casasnovas O, Petrella T, et al: Molecular profiling of classical Hodgkin lymphoma tissues uncovers variations in the tumor microenvironment and correlations with EBV infection and outcome. Blood 113: 2765-3775, 2009

16. Agueli C, Cammarata G, Salemi D, Dagnino L, Nicoletti R, La Rosa M, Messana F, Marfia A, Bica MG, Coniglio ML, et al: $14 \mathrm{q} 32 / \mathrm{miRNA}$ clusters loss of heterozygosity in acute lymphoblastic leukemia is associated with up-regulation of BCL11a. Am J Hematol 85: 575-578, 2010.
17. Jiang BY, Zhang XC, Su J, Meng W, Yang XN, Yang JJ, Zhou Q, Chen ZY, Chen ZH, Xie Z, et al: BCL11A overexpression predicts survival and relapse in non-small cell lung cancer and is modulated by microRNA-30a and gene amplification. Mol Cancer 12: 61, 2013.

18. Lazarus KA, Hadi F,Zambon E, Bach K, Santolla MF, Watson JK, Correia LL, Das M, Ugur R, Pensa S, et al: BCL11A interacts with SOX2 to control the expression of epigenetic regulators in lung squamous carcinoma. Nat Commun 9: 3327, 2018.

19. Zhang X, Wang L, Wang Y, Shi S, Zhu H, Xiao F, Yang J, Yang A and Hao X: Inhibition of FOXQ1 induces apoptosis and suppresses proliferation in prostate cancer cells by controlling BCL11A/MDM2 expression. Oncol Rep 36: 2349-2356, 2016.

20. Kaneda H, Arao T, Tanaka K, Tamura D, Aomatsu K, Kudo K, Sakai K, De Velasco MA, Matsumoto K, Fujita Y, et al: FOXQ1 is overexpressed in colorectal cancer and enhances tumorigenicity and tumor growth. Cancer Res 70: 2053-2063, 2010.

21. Zhu L, Pan R, Zhou D, Ye G and Tan W: BCL11A enhances stemness and promotes progression by activating Wnt/ $\beta$-catenin signaling in breast cancer. Cancer Manag Res 11: 2997-3007, 2019.

22. He D, Wu H, Ding L and Li Y: Combination of BCL11A siRNA with vincristine increases the apoptosis of SUDHL6 cells. Eur J Med Res 19: 34, 2014

23. Gao Y, Wu H, He D, Hu X and Li Y: Downregulation of BCL11A by siRNA induces apoptosis in B lymphoma cell lines. Biomed Rep 1: 47-52, 2013.

24. Yang F, Shen Y, Zhang W, Jin J, Huang D, Fang H, Ji W, Shi Y, Tang L, Chen W, et al: An androgen receptor negatively induced long non-coding RNA ARNILA binding to miR-204 promotes the invasion and metastasis of triple-negative breast cancer. Cell Death Differ 25: 2209-2220, 2018.

25. Zhu A, Li Y, Song W, Xu Y, Yang F, Zhang W, Yin Y and Guan X: Antiproliferative effect of androgen receptor inhibition in mesenchymal Stem-like triple-negative breast cancer. Cell Physiol Biochem 38: 1003-1014, 2016.

26. Zhang W, Luo J, Yang F, Wang Y, Yin Y, Strom A, Gustafsson J and Guan X: BRCA1 inhibits AR-mediated proliferation of breast cancer cells through the activation of SIRT1. Sci Rep 6: 22034, 2016.

27. Ji W, Shi Y, Wang X, He W, Tang L, Tian S, Jiang H, Shu Y and Guan X: Combined androgen receptor blockade overcomes the resistance of breast cancer cells to palbociclib. Int J Biol Sci 15: $522-532,2019$

28. Sankaran VG, Xu J, Ragoczy T, Ippolito GC, Walkley CR, Maika SD, Fujiwara Y, Ito M, Groudine M, Bender MA, et al: Developmental and species-divergent globin switching are driven by BCL11A. Nature 460: 1093-1097, 2009.

29. Lin Y, Zhang Q, Zhang HM, Liu W, Liu CJ, Li Q and Guo AY: Transcription factor and miRNA co-regulatory network reveals shared and specific regulators in the development of B cell and T cell. Sci Rep 5: 15215, 2015.

30. Hayashi N, Manyam GC, Gonzalez-Angulo AM, Niikura N, Yamauchi H, Nakamura S, Hortobágyi GN, Baggerly KA and Ueno NT: Reverse-phase protein array for prediction of patients at low risk of developing bone metastasis from breast cancer. Oncologist 19: 909-914, 2014.

31. Tsang JC, Yu Y, Burke S, Buettner F, Wang C, Kolodziejczyk AA, Teichmann SA, Lu L and Liu P: Single-cell transcriptomic reconstruction reveals cell cycle and multi-lineage differentiation defects in Bcl11a-deficient hematopoietic stem cells. Genome Biol 16: 178, 2015.

32. Wiegreffe C, Simon R, Peschkes K, Kling C, Strehle M, Cheng J, Srivatsa S, Liu P, Jenkins NA, Copeland NG, et al: Bcl11a (Ctip1) controls migration of cortical projection neurons through regulation of Sema3c. Neuron 87: 311-325, 2015.

This work is licensed under a Creative Commons Attribution-NonCommercial-NoDerivatives 4.0 International (CC BY-NC-ND 4.0) License. 